\title{
Sommes-nous toujours honnêtes dans nos pratiques de la recherche?
}

\begin{abstract}
"The description of a scientist as honest should be a tautology" conclut un article récent de Nature consacré à la fraude en recherche. Peut-on penser qu'il pourrait en être autrement ? La fiabilité des résultats de la recherche, la rigueur et l'honnêteté de leur interprétation sont essentiels pour l'avancement de la connaissance et pour garantir la confiance du public dans l'entreprise de la recherche. Pourtant, on parle de plus en plus de cas de plagiat, de données falsifiées voire inventées. La proportion d'articles rétractés augmente considérablement (fig. 1), dont $43 \%$ pour fraude, comme le souligne en 2012 la prestigieuse revue $P N A S^{(a)}[1]$. Il ne s'agit d'ailleurs pas d'un phénomène nouveau puisqu'il a concerné, comme l'on sait, de grands scientifiques tels que Mendel ou Pasteur et Galilée, Newton ou Millikan. Pourtant il semble s'amplifier, devient un motif croissant d'inquiétude pour les institutions de recherche et fait l'objet depuis quelques années de conférences mondiales portant sur l'intégrité en science et aboutissant à des chartes.
\end{abstract}

Le domaine des sciences de la vie et de la santé semble le premier concerné. C'est en effet une science beaucoup plus jeune que les mathématiques ou la physique, et qui traite de sujets d'une infinie complexité. En outre, le financement de la recherche médicale par des laboratoires privés peut prédisposer aux conflits d'intérêt, voire à la corruption. Un exemple célèbre est celui de Scott Reuben, anesthésiste britannique réputé, financé par la firme Pfizer, qui publia vingt-et-un articles de 1995 à 2006, avec des résultats totalement inventés sur l'efficacité d'un médicament agissant sur les douleurs postopératoires. Plus grave, celui d'Andrew Wakefield démontrant faussement que le vaccin contre la rougeole prédispose à l'autisme, entraînant une diminution importante de la couverture vaccinale en Angleterre. Mais la fraude concerne aussi bien d'autres domaines de la science. La sociologie, sans même évoquer l'économie, fournit de nombreux cas choquants, tel celui bien connu de Cyril Burt qui mit en évidence de très fortes corrélations statistiques entre les QI de jumeaux homozygotes totalement séparés peu après leur naissance ; ces études se sont révélées truquées, mais ont eu des conséquences durables sur les pratiques éducatives en Grande-Bretagne.

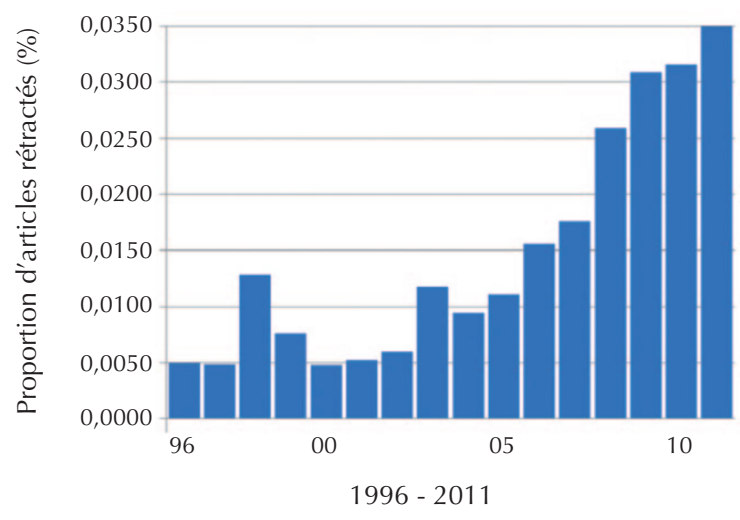

1. L'évolution des rétractations dans la littérature biomédicale, de 1996 à 2011, d'après l'Office of Research Integrity (mars 2013). Ce nombre est rapporté au nombre total d'articles (indexés dans MEDLINE) parus la même année. Les données les plus récentes pour 2012 (absentes de ce graphique) sont de 348 rétractations pour 760000 articles publiés, soit 0,0457\%, ou une augmentation de $30,5 \%$ par rapport à 2011. D’après R. van Noorden [2], 44\% des rétractations relèvent d'une " mauvaise conduite " (falsification, plagiat ou autoplagiat)

Si les mathématiques semblent relativement à l'abri des pratiques frauduleuses, qu'en est-il de la physique ? L'affaire de Hendrick Schön nous rappelle qu'elle non plus n'y échappe pas. Ce physicien réputé des Bell Labs, entouré d'une équipe de vingt collaborateurs, ambitionnait de révolutionner la supraconductivité, l'électronique moléculaire, les transistors et les nanotechnologies. En 2000, il publia huit articles dans Science et Nature, en 2001 un article tous les huit jours ! Une commission d'experts finit par conclure, en dépit de la destruction des cahiers d'expériences et des échantillons, qu'il avait délibérément falsifié, voire inventé, des données expérimentales. Schön a certes été confondu, mais que penser de ses collaborateurs exonérés de toute accusation et du filtrage déficient par le "peer review" des journaux scientifiques?

La fraude avérée reste tout de même exceptionnelle ; les quelques cas signalés au CNRS sont traités dans la plus grande discrétion au niveau de la direction générale, après vérification des allégations par un comité ad hoc. Par contre, ce qui paraît plus préoccupant, c'est le glissement actuel, conscient ou inconscient, de certains of the National

Academy of Sciences of the United States of America. 
chercheurs vers des conduites éthiquement discutables. Et il y a une continuité entre la fraude avérée et les résultats arrangés : on "cuisine " des données pour ne garder que les points qui "collent "; on «trafique " des clichés de manip avec Photoshop ; on publie trop vite des résultats qu'on n'a pas réussi à reproduire ; on reste évasif sur les protocoles expérimentaux pour ne pas être vérifié ou copié ; on dissimule des résultats ; on «saucissonne » ses données en plusieurs articles au risque de rendre incompréhensible chacun pris isolément, etc. Le domaine des signatures des articles est bien celui qui suscite le plus de comportements peu intègres et donc de conflits dans une équipe ou même d'un laboratoire à l'autre : on "oublie " de mettre sur la publication le nom du postdoctorant reparti dans son pays ; à l'inverse, on cite par complaisance celui du grand patron ; on joue à "je te cite, tu me cites "; on laisse publier des résultats douteux à un doctorant pressé de boucler sa thèse ; on se querelle sur l'ordre supposé significatif des signataires ; on emprunte des données à des chers collègues sans leur demander leur accord. Pire : on vole une idée à un projet dont on a été rapporteur dans un comité d'évaluation, impunément car il n'y a pas de droit de propriété intellectuelle sur les idées non publiées...

Dans un autre registre, certains chercheurs n'ont pas une pleine conscience de leurs responsabilités dans le travail collectif. Gérer les relations hiérarchiques, savoir respecter ses collaborateurs, reconnaitre leur contribution peut se révéler complexe et délicat, d'autant qu'il n'existe quasiment pas de formation au management des personnels dans un laboratoire. La responsabilité des directeurs de thèse dans la formation des doctorants, telle que définie dans la charte des thèses, se révèle assez souvent mise en défaut par manque de suivi ; il importe en effet de bien les initier avec rigueur aux méthodes de la discipline, de les former à l'écriture d'articles, de leur permettre l'accès à des congrès, enfin de leur apporter une aide pour la suite de leur carrière. À côté de conseils insuffisants aux étudiants et d'ailleurs aussi aux postdoctorants, tous dans une situation de précarité, l'abus d'autorité et le harcèlement sont fréquents dans les laboratoires. Certes les discriminations et le harcèlement, moral ou sexuel, relèvent du droit du travail et peuvent être par une jeune doctorante contre son directeur de thèse, ou par un chercheur novice exploité par son patron, au risque de détruire ses perspectives de carrière dans un milieu où tout le monde se connaît et se soutient à une certain niveau ?

À quoi attribuer ces dérives, mineures ou graves, dont se rend coupable un chercheur sur trois (si l'on en croit une enquête publiée dans Nature en 2011 [2]) ? Inutile de revenir ici sur la pression pour publier qui est devenue la règle, avec la dictature imparable de la bibliométrie [3]. Les scientifiques sont de plus en plus confrontés aux profondes mutations de la recherche et à l'évolution de leurs missions, dans un contexte très fortement compétitif avec l'internationalisation de la recherche et les pressions de l'évaluation qui conditionne l'évolution des carrières. Ils doivent valoriser leurs travaux, trouver des financements, prendre en charge des expertises, diffuser la culture scientifique et enfin assurer une communication avec le public et les médias. Notons que certains ont aussi de lourdes charges d'enseignement. Concilier la passion de découvrir et de contribuer au bien commun avec cet ensemble de contraintes, relève d'un défi permanent et rend les chercheurs vulnérables à des écarts.

En France, une charte nationale relative à l'intégrité scientifique est en cours d'élaboration. Le comité d'éthique du CNRS (le COMETS) vient de publier un guide intitulé "Promouvoir une recherche intègre et responsable » [4], qui a vocation à s'étendre à toutes les institutions de recherche en France.

Mais comme l'a écrit Pierre Léna, ancien président de la Société Française de Physique et aussi du COMETS : "On ne peut multiplier des barrières, les interdits, les règlements... dans bien des cas, c'est à la conscience du chercheur que l'on fait appel."

"Hippocrate a écrit un serment que chaque médecin prête depuis cinq siècles. N'est-il pas temps de penser à un serment du scientifique ?" (Pierre Léna et Michel Serres).

Michèle Leduc ${ }^{(1)}$ (michele.leduc@lkb.ens.fr)

Reflets de la physique n'32 (janvier 2013), p. 46.

$4 \bullet$ www.cnrs.fr/comets/ IMG/pdf/guide_final_ 19-11-2013.pdf sanctionnés. Mais qui imagine une plainte déposée

\section{Références}

F. Fang et al.,
"Misconduct accol retracted publications",

17028-33.

"The trouble with retraction", Nature,

78 (2011) 26-28.

\section{et Lucienne Letellier
$(2)$
recherche émérite au Laboratoire Kastler-Brossel}

(1) Directrice de recherche émérite au Laboratoire Kastler-Brossel
(ENS et UPMC), présidente du comité d'éthique du CNRS (COMETS)

(2) Directrice de recherche émérite à l'IBBMC (Université Paris-Sud), experte associée au COMETS 